\title{
Module of System Galactica with Coulomb's Interaction
}

\author{
Joseph J. Smulsky \\ Institute of Earth Cryosphere SB RAS, 625000, Tumen, P.O.Box 1230, Russia \\ Email: jsmulsky@mail.ru
}

\begin{abstract}
The system Galactica of free access is supplemented module for the Coulomb interaction. It is based on a high-precision method for solving differential equations of motion of $N$ charged particles. The paper presents all the theoretical and practical issues required to use this module of system Galactica so that even the beginning researcher could study the motion of particles, atoms and molecules.
\end{abstract}

Index Terms-Coulomb Interactions, Equations of Motion, Computer Simulations, Models of Atoms, Free Access.

\section{INTRODUCTION}

System Galactica for gravitational interaction of bodies in the form of material points was created in the beginning of 2012 [1]. It is in free access [2]. Then ideas of making similar systems for other interactions had appeared. As their structure is similar to system Galactica, it has been decided to make them in the form of additional modules for system Galactica. They will be added in description by separate sections, and in the file system by new folders. The Module of System Galactica with Coulomb's Interaction is represented in section 14. Module of system Galactica with Coulomb's interaction [2]. Till section 14 the manual of system Galactica with gravitational interaction between material-point bodies is given.

In physics the Coulomb's interaction usually is considered on the basis of the two-body problem. In quantum mechanics behavior of ensembles of particles and their properties are studied as a result of statistical processing of two-partial interactions. Together with such quantum-mechanical consideration of the microworld, a number of researchers continue to apply classical mechanics for explanation of a series of its phenomena. A.D. Vlasov [3] in his researches has concluded about justice of the classical electrodynamics laws in atom and about inability of probabilistic interpretation of atomic phenomena. F.M. Kanarev [4]-[5] explains atoms radiation spectra on the basis of classical physics. During several decades M. Gryziński consistently considers the microworld phenomena, based on the Coulomb's mechanism of interaction. For instance, he explains diffraction phenomena by electron spin precession [6]. Using binary Coulomb's interactions, M. Gryziński explains single and double ionization, radiation of one or triplet lines, diffraction of particles at their dispersion on atoms and molecules [7]. He has showed that taking into account of action of atom electron shell explains Ramsauer's effect about low dispersion of electrons at their low energy [8]-[9]. On the basis of classical mechanics M. Gryziński has received equations for determination of absolute braking energy of particles by any medium in all nonrelativistic spectrums of energies [10].

The above-mentioned researchers obtained the enumerated results by analytical methods. However, all these tasks are difficult, and only separate problems of many particles interaction can be solved even at high mathematical level of a researcher. Therefore use of highprecision numerical methods of solution of these problems of Coulomb's interaction opens a prospect of determined knowledge of the micro-world.

As shown in works [11], [12], [13], the essential change of particles trajectories happens at velocities of motion $v>0.1 c$, where $c$ is speed of light. Therefore it is possible to investigate the phenomena unto velocities of this order by system Galactica with Coulomb's interaction. It is necessary to use exact expression for the force of electromagnetic interaction of two particles [11] in the case of higher velocities. Further we hope update system Galactica by the module for calculation of electromagnetic interactions at high velocities which are now called relativistic ones.

\section{Essentials Of The Module}

The module is intended for solving problems in mechanical interaction of material-point particles according to the Coulomb's law. Module files are located in folder GalactcW/ModCoulm [2]. This folder contains other folders: InCndFsQ, InpFilsQ and RunFilsQ. The folder GalactcW contains files GalDiscrp.pdf and GalDiscrE.pdf that are manual of Galactica respectively in Russian and in English. The zipped folders can be downloaded at

http://www.ikz.ru/ smulski/GalactcW/ModClFlds.zip.

The system includes named executable files, for instance, glk3b30e, to be run on a supercomputer under Unix OS, or glk3b30.exe, to be run under DOS; an input file galacf26.dat, and a named initial data file specifying the interacting bodies and the initial conditions of their motion, for instance, axsyk109.dat. Below, the latter file will be referred to as the initial condition (IC) file. 
The results yielded by the executable file are produced as output files. The representation form is defined by keys specified in the input file galacf26.dat. Following a certain number of steps, characteristics of body motions can be issued; files containing such data are named 1, 2, 3... garek29.dat. The file garek29.dat is always produced on completion of the program. This file contains characteristics of bodies at the final calculation step. This is the main form of problem solution output.

Besides, if certain keys are set in the input file galacf26.dat, then parameters defining the trajectory of particles, whose numbers are also defined in the input file, are issued too. The names of the trajectory files are as follows: $1 \mathrm{t}, 2 \mathrm{t}, 3 \mathrm{t}$... traekt.prn. The file size is defined by the volume of the data set in the input file.

If certain keys are set, then a file dice.dat is issued. The file contains data on the time of close approach of bodies, minimal distance between bodies, and the number of a body approached by the given body. Each line in the file dice.dat is formed on accomplishment of a certain number of integration steps. The number of such steps is specified in the file galacf26.dat.

With a certain key set, a binary file (or, depending on the particular computer, an unformatted file) named galgok2 is issued. This file contains the state of the problem in binary form at the last calculation step. On subsequent start of the program, calculation is continued starting from this step, the data contained in the input file and in the initial condition file not being read out. The latter procedure is required in solving problems with long running times. The interruptions in calculations do not worsen the solution accuracy.

In the case of an emergency situation, an error file ErrGlctca is generated; this file contained a description of the error having occurred. Such errors can be encountered in opening files and their reading, and they also can be a result of insufficient array dimensions or impossibility to meet accuracy requirements in calculation of bodies merging together on collision.

In Galactica, the possibility to produce a screen of data is provided. Those parameters common to all bodies allows one to monitor both the execution of the program and the calculation accuracy. In solving a problem on a supercomputer, such data are saved in an output stream file.

In a version of Galactica with PC display graphics, along with common problem parameters, images of bodies with their velocity vectors can be produced. The form of images is defined by keys and parameters set in the input file galacf26.dat.

\section{DifFERENTIAL EQUATIONS OF MOTION FoR MATERIAL-POINT PARTICLES}

Let there is a system of $N$ material points (particles) with masses of $m_{i}$ and electrical charges $q_{i}$, where $i=1$, 2 .. $N$. Let's designate dimensional coordinates and velocities of particle $i$ as $x_{m i}, y_{m i}, z_{m i}, v_{x m i}, v_{y m i}, v_{z m i}$ in the non-accelerated system of coordinates relative to the center of mass $C$. In particular, the coordinates can be expressed in meters and velocities in $\mathrm{m} / \mathrm{sec}$. A particle with number $k$ acts on $i$-th particle by the electrostatic action, which will be written down as Coulomb's force projection onto the axis $x_{m}$ so:

$$
F_{x i k}=\frac{q_{i} q_{k}\left(x_{m i}-x_{m k}\right)}{\varepsilon_{d} \cdot r_{m i k}^{3}},
$$

where $\varepsilon_{d}$ is dielectric permeability of medium, in which the particles are situated;

$$
r_{m i k}=\sqrt{\left(x_{m i}-x_{m k}\right)^{2}+\left(y_{m i}-y_{m k}\right)^{2}+\left(z_{m i}-z_{m k}\right)^{2}}
$$

is the distance between the $i$-th and $k$-th particles.

The expression (1) for force projection onto the axis $x_{m}$ is written down in CGS electrostatic system. The formula for force projection onto the axes $y_{m}$ and $z_{m}$ looks similarly. Therefore all expressions will be written down here and below for one projection. With summation of all particles forces (1), the Coulomb's force of their action upon the particle with number $i$ will be

$$
F_{x i}=q_{i} \sum_{k \neq i}^{N} \frac{q_{k}\left(x_{m i}-x_{m k}\right)}{\varepsilon_{d} r_{m i k}^{3}},
$$

where summation for $k=1,2 \ldots N$, except $k=i$, is designated by symbol $\Sigma$.

Under the action by force (3), in accordance with the second law of mechanics, the particle $i$-th will set in motion relative to a non-accelerated reference system with acceleration

$$
\frac{d v_{m x i}}{d t}=\frac{q_{i}}{m_{i}} \sum_{k \neq i}^{N} \frac{q_{k}\left(x_{m i}-x_{m k}\right)}{\varepsilon_{d} r_{m i k}{ }^{3}}
$$

Below the problem is solved in non-dimensional form. Characteristic size $\mathrm{Am}$ of an area, in which there are electric particle, is set for it. All charges $q_{i}$ are reduced by absolute value of electron charge $e_{e}$, which $e_{e}=$ $+4.80298 \cdot 10^{-10} \mathrm{~cm}^{3 / 2} \cdot \mathrm{g}^{1 / 2} \cdot \mathrm{sec}^{-1}$, for instance, in CGS electrostatic system. The masses $m_{i}$ are reduced by total mass $M_{S S}$ of all system of interacting particles. Then equation (4) will look in non-dimensional form so:

$$
\frac{d v_{x i}}{d T}=-q_{m i} \cdot \sum_{k \neq i}^{N} \frac{q_{o k} \cdot\left(x_{i}-x_{k}\right)}{R_{i k}{ }^{3}}, i=1,2, \ldots, N,
$$

where $x_{i}=x_{m i} / A m$;

$$
\begin{aligned}
& R_{i k}=\sqrt{\left(x_{i}-x_{k}\right)^{2}+\left(y_{i}-y_{k}\right)^{2}+\left(z_{i}-z_{k}\right)^{2}} ; \\
& q_{o i}=q_{i} / e_{e} ; \quad q_{m i}=-q_{o i} / m_{o i} ; \\
& m_{o i}=m_{i} / M_{S S} ; \quad M_{S S}=\sum_{i=1}^{N} m_{i} ;
\end{aligned}
$$




$$
\begin{aligned}
& v_{x i}=v_{m x i} \cdot k_{v} ; \quad k_{v}=\sqrt{\frac{\varepsilon_{d} M_{S S} \cdot A m}{e_{e}^{2}}} ; \\
& T=t \cdot k_{t} ; \quad k_{t}=\sqrt{\frac{e_{e}^{2}}{\varepsilon_{d} \cdot M_{S S} \cdot A m^{3}}}=1 /\left(A m \cdot k_{v}\right)
\end{aligned}
$$

Equation (5) for three projections $x, y$ and $z$ represents $3 \cdot N$ second order differential equations, which determine motion of the charged particles. Non-dimensional charges $q_{o i}$ of particles can be positive and negative. Value of non-dimensional variables, which describe motion by equations (5), depends on arbitrary parameter $\mathrm{Am}$. Its value can be chosen such that non-dimensional time $T$ has been in convenient units for consideration of interactions in the microworld.

If the primary scale is time coefficient $k_{t}$, then the scale length $A m$ entering the expression (10) for $k_{t}$ can be calculated as

$$
A m=\left(\frac{e_{e}^{2}}{\varepsilon_{d} \cdot M_{S S} \cdot k_{t}^{2}}\right)^{1 / 3}
$$

Gravitational interactions in program Galactica are determined by the following non-dimensional equation (6) in [1]. Let's copy it without changes:

$$
\frac{d v_{x i}}{d T}=-\sum_{k \neq i}^{N} \frac{m o_{k}\left(x_{i}-x_{k}\right)}{r_{i k}{ }^{3}}, i=1,2, \ldots, N
$$

It is visible from comparison (5) with (12) that the algorithm for gravitational interaction can be adapted to Coulomb's one if, first, to replace $m o_{k}=m_{o k}$ with $q_{o k}$ and, secondly, to multiply expression (12) for acceleration $d v_{x i} / d T$ by $q_{m i}$. These changes were done in program Galactica, and Fortran-program glk3pb30.for for Coulomb's interaction is developed [14].

\section{Identical Properties Of The Module}

In this section, we note the identical properties of the module for Coulomb interaction with components of the system Galactica for the gravitational interaction.

The method of solving equations (4) remains the same as approach for the equations of the gravitational interaction (12). The same input file galacf26.dat is used [1]. The submitted opportunities in item 5.2 its description [1] for the Coulomb interaction are not used. The files of trajectories $1 \mathrm{t}, 2 \mathrm{t} \ldots$ traekt.prn and file encounters dice.dat have the same content as that of a gravitational interaction.

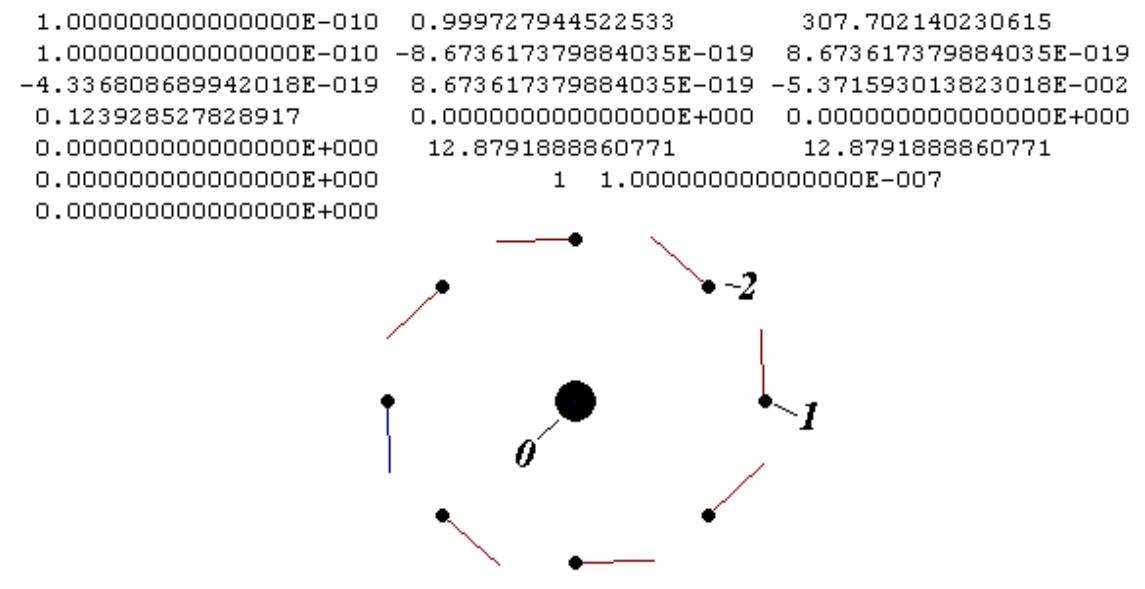

Fig. 1. Axisymmetric Coulomb's interaction between 9 particles with parameters for oxygen atom: 0 - the central particle; 1 - the first peripheral particle; 2 - the second peripheral particle. Case of circular motion.

Similarly Fig. 1 in section 8 for gravitational interaction [1], Fig. 1 yields output for Coulomb's interaction [14]. In solving a problem on a PC with a Galactica program with graphic option, numerical data concerning the integration process and a picture illustrating the position of particles with their velocity vectors are given out to the display screen following a certain number of integration steps (see Fig. 1). The data allow one to monitor the solution process and to observe solution reliability. In using a no-graphics version of Galactica, only numerical data are output to the screen. In solving the problem on a supercomputer, the data are issued to the output stream file. The description of numerical information is available in section 9 [1].

\section{INITIAL CONDITION AND INTEGRATION DATA FILES}

This section is based on section 5 [1] for gravitational interaction. Some differences for Coulomb's interaction are given below.

Each of initial condition files, for instance, axsyk109.dat in folder InCndFsQ, and also output files: 1, 2, 3, etc. and file garek29.dat begin with a set of 25 
numbers: T0, omm, Um, dTp, Px, Py, Pz, AMx, AMy, AMz, Spsx, Spsy, Spsz, E, Em, Ett, dT, i2b, j2b, k2b, A, $\mathrm{B}, \mathrm{C}, \mathrm{Mu}, \varepsilon \mathrm{d}$. Those numbers are common to all interacting particles. Then, sixteen numbers for each $\mathrm{k}$-th particle follow: $\mathrm{om}(\mathrm{k}),(\mathrm{X}(\mathrm{k}, \mathrm{q}), \mathrm{q}=1,3),(\mathrm{U}(\mathrm{k}, \mathrm{q}), \mathrm{q}=1,3)$, $(\mathrm{dUp}(\mathrm{k}, \mathrm{q}), \mathrm{q}=1,3),(\mathrm{Sp}(\mathrm{k}, \mathrm{q}), \mathrm{q}=1,3), \operatorname{Ra}(\mathrm{k}), \operatorname{Et}(\mathrm{k}), \mathrm{qo}(\mathrm{k})$. Here, $\mathrm{q}$ is the subscript listing the variables over the three coordinates $\mathrm{x}, \mathrm{y}$, and $\mathrm{z}$.

The general parameters represent the following:

T0 - time in non-dimensional units;

omm - maximal mass of body reduced by the total mass of all particles;

Um - maximal velocity in non-dimensional units;

$\mathrm{dTp}$ - time step in non-dimensional units at the previous step;

$\mathrm{Px}, \mathrm{Py}, \mathrm{Pz}-$ projections of the total momentum of the whole particle system on the coordinate axes;

$\mathrm{AMx}, \mathrm{AMy}, \mathrm{AMz}$ - projections of the total angular momentum of the whole particle system;

Spsx, Spsy, Spsz - projections of the sum of the angular momenta due to self-rotation of bodies around their axes (spin projections);

$\mathrm{E}$ - total kinetic energy of all particles at the given moment T0;

Em - maximal kinetic energy of all particles starting from the beginning of integration;

Ett - thermal energy of all bodies which the particles have acquired during their merging on collision;

$\mathrm{dT}$ - current time step in non-dimensional units;

$\varepsilon \mathrm{d}-$ dielectric permeability of medium, which there are the particles in.

Next, 16 parameters for each particle follow:

om(k) $\div m_{o \mathrm{k}}-$ non-dimensional mass of the $\mathrm{k}=i$-th particle ( $\mathrm{k}$ and $i$ are the numbers of particles in different texts);

$(\mathrm{X}(\mathrm{k}, \mathrm{q}), \mathrm{q}=1,3) \div x_{i}, y_{i}, z_{i}-$ non-dimensional barycentric coordinates of the $\mathrm{k}=i$-th particle;

$(\mathrm{U}(\mathrm{k}, \mathrm{q}), \mathrm{q}=1,3) \div v_{x i}, \quad v_{y i}, \quad v_{z i}-$ non-dimensional velocities of the $\mathrm{k}=i$-th particle;

$(\mathrm{dUp}(\mathrm{k}, \mathrm{q}), \mathrm{q}=1,3) \div x_{i}^{(5)}, y_{i}^{(5)}, z_{i}^{(5)}-$ non-dimensional derivatives of the fifth order for the $\mathrm{k}=i$-th particle;

$(\operatorname{Sp}(\mathrm{k}, \mathrm{q}), \mathrm{q}=1,3) \div S_{p x i}, \quad S_{p y i}, \quad S_{p z i}-$ non-dimensional angular momenta (spins) due to the self-rotation of the $\mathrm{k}$ $=i$-th particle. A particle is assumed to get a spin if the particle has formed as a result of the merge of other particles having approached each other to a distance smaller than the sum of their radii. Initially, the rotation of particles can be ignored, with their spins being defined as $S_{p x i}=S_{p y i}=S_{p z i}=0$. This strategy is adopted in the initial condition files given below.

$\mathrm{Ra}(\mathrm{k})$ - non-dimensional equivalent radius of the $\mathrm{k}=i$ th particle;

$\operatorname{Et}(\mathrm{k})-E_{t i}-$ non-dimensional thermal energy of the $\mathrm{k}=$ $i$-th particle. This energy has arisen from merging of several particles in one particle due to transformation of kinetic energy into thermal energy.

$\mathrm{qo}(\mathrm{k})$ - non-dimensional charge of the $\mathrm{k}=i$-th particle.

In Appendix 1 there is the sample of program InCnPrClb.med in MathCad software for generation of initial condition file.

\section{EXAMPles Of InPUT Files GALACF26.DAT AND IC- FILES}

Section 10 [1] gives samples of input files for different problems of gravitational interaction. These samples show functions of various parameters of file galacf26.dat.

The folder InpFilsQ contains example of input file galacf26.dat, which was used for axisymmetric Coulomb's interaction of 9 particles (see Fig. 1) in work [14]. This folder also contains input files with changed names g26_2ptk, g26_3ptk, g26_175ptk, g26_473ptk for problems with $2,3, \overline{175}$ and $4 \overline{73}$ particles. Files with 2 and 3 particles were used in work [14]. The force of attraction between a peripheral particle and the central particle is maximal for axisymmetric configuration with 175 particles, and minimal for one with 473 particles.

Section 11 [1] involves initial condition files for various problems of gravitational interaction. The folder InCndFsQ contains file ax8yk109.dat for axisymmetric Coulomb's interaction of 9 particles. Thus 8 peripheral particles move along elliptic orbits with eccentricity $e=$ 0.15 . This folder also contains initial condition files with analogical names for interaction of 2, 3, 175 and 473 particles. Their orbits are circles. The program InCnPrClb.mcd, contained in this folder, is intended for creation of such initial condition files.

\section{EXAMPLES Of INPUT Files GALACF26.DAT AND IC- FILES}

In section 12 [1] the executable files, which are in the folder RunFiles, are described in the case of gravitational interaction. The folder RunFilsQ contains executable files in the case of Coulomb's interaction. Table 1 lists the names of the main exe-files for PC and for a Unix OS supercomputer. In the names, the following agreements are adopted. Symbols glk mean Galactica with Coulomb's interaction. Digit 3 means a third version of the program intended for common use. The character «b» with subsequent symbols means the total number of bodies (particles).

Executable files without graphics compiled by Fortran PowerStation v4.0. Codes with graphics, which are compiled by the Compaq Visual Fortran Professional Edition v6.5.0 in Fortran standard graphics, have in a name the letter $\mathrm{p}$.

Table 1. NAMES OF EXE-FILES OF GALACTICA WiTH COULOMB'S INTERACTION

\begin{tabular}{|c|c|c|c|}
\hline \multirow{2}{*}{$\begin{array}{l}\text { Number of } \\
\text { interacting } \\
\text { particles }\end{array}$} & \multicolumn{2}{|c|}{ For PC } & \multirow{2}{*}{$\begin{array}{c}\text { For a UNIX } \\
\text { OS } \\
\text { supercomputer }\end{array}$} \\
\hline & $\begin{array}{l}\text { Without } \\
\text { graphics }\end{array}$ & With graphics & \\
\hline 30 & - & - & glk3b30e \\
\hline 300 & glk3b3c.exe & glk3pb3c.exe & - \\
\hline 3000 & glk3b3t.exe & glk3pb3t.exe & glk3b3t \\
\hline
\end{tabular}

The code named glk3b30e capable of calculating 30 particles on a supercomputer operates with extended digit length (34 decimal symbols). This program can read initial condition files with double length numbers, and it 
generates integration data files with digits of quadruple length. The speed of calculation with this program is 33 times lower in comparison with other codes. All codes except for the latter one use double length numbers.

\section{GENERATION OF INITIAL CONDITION FILE}

In section 13 [1] the recommendations for generation of initial condition file, the part of which can be used in the case Coulomb's interaction, are given in the case of gravitational interaction. As it is noted in section 13, there are features of algorithm for calculating initial conditions for each task. As applied to the problem of axisymmetric Coulomb's interaction [14], the file InCnPrClb.mcd (see Appendix 1) exemplifies a procedure for generation of an initial condition file in MathCad environment. This sample can be implemented using any programming language or mathematical software. Below, taking the sample as an example, we will comment on the particular actions. In the problem of interest, the following configuration of interacting particles is defined: around a central particle, peripheral particles are located. The velocities of the particles are chosen such that the particles move along elliptic orbits with eccentricity $e$. The central particle has a positive charge, and peripheral ones have a negative charge. Such problem has a exact analytical solution. In works [11], [12], [15] and [16] it is given for gravitational interaction, and in work [14] - for Coulomb's one. Such axisymmetric configurations are unstable at certain parameters. Therefore in the case of numerical solution of the particles interaction equations, their dynamic features are determining. In work [14] the interaction between the central particle and eight and two peripheral particles, and also interaction between two particles are considered. The program InCnPrClb.med was used for generation of initial condition file for these tasks. Here it also was used for generation of initial condition file for these tasks to the number of particles 175 and 473. The description of the basic operations on items of the program is given below. The numbers of operations are starting with the number 14.12, which means the following. In the manual of the system Galactica [2] the module with Coulomb interaction, as noted at the beginning, is follow in section 14, and the item in question is number 12 .

Item 14.12.1 of Appendix 1 defines the total number of interacting bodies $\mathrm{Nb}$ and the lengths of indexes $\mathrm{i}$ and $\mathrm{i} 1$. Since an index begins with zero, the largest value of $\mathrm{N}$ is smaller than $\mathrm{Nb}$ by unity.

Item 14.12.2 defines according to [17] the main constants, namely, masses of electron $m_{e}$, proton $m_{p}$ and neutron $m_{n}$ in $\mathrm{kg}$; charge of electron $e_{e}$ in CGS electrostatic system; base radius $R_{0}$, which atom nucleus radius is determined by

$$
R_{n}=R_{0} \cdot A_{N}^{1 / 3}
$$

where $A_{N}$ is mass number of an atomic nucleus.
Besides, the item 14.12.2 defines electron radius $R_{e}$, dielectric permeability of medium $\varepsilon_{d}$ in CGS electrostatic system and the first Bohr's orbit radius $a_{B 0}$, by which the average distance between electron and nucleus (see $\mathrm{p}$. 749 [17]) is determined:

$$
r_{m}=\frac{a_{B o}}{2 \cdot Z_{p}}\left[3 \cdot n_{n}^{2}-l_{n}\left(l_{n}+1\right)\right]
$$

where $Z_{p}$ is charge of an atomic nucleus;

$n_{n}$ and $l_{n}$ are quantum numbers.

Item 14.12.3 defines axisymmetric configuration with 8 peripheral particles by the example of oxygen atom, including atomic number $A_{N}$, nucleus charge $Z_{p}$ and quantum numbers $n_{n}$ and $l_{n}$ for determination of average distance $a=r_{m}$ between peripheral electrons ring and nucleus by formula (14). This item also defines the orbits eccentricity of peripheral particles and the plane inclination $\varepsilon_{0}$ of their orbits $x_{0} y_{0}$ in the coordinate system $x y z$, for which the equations (5) are written. The orbits plane $x_{0} y_{0}$ is revolved through an angle $\varepsilon_{0}$ relative to the axis $x$.

In item 14.12.4 the masses of the central particle $m_{0}$ and the peripheral particles $m_{i 1}$ in kilograms, and also their radii $\mathrm{ram}_{0}$ and $\mathrm{ram}_{i 1}$ in meters are calculated.

Then, in item 14.12.5 the algorithm of [1], [11], [12], [15] is implemented to determine the coordinates and velocities of the peripheral particles in the orbit plane. Here, the notation introduced in our book «The Theory of Interaction» [11] - [12] is used: $\alpha_{1}$ is the trajectory parameter, $\mu_{1}$ is the interaction parameter, and the pericenter parameters are $R_{p}$ and $v_{p}: R_{p}$ is the radius and $\mathrm{v}_{\mathrm{p}}$ is velocity.

This algorithm is intended for calculating the interaction of an arbitrary number of particles. That is why one has to check that the orbit will not be overcrowded with the particles. This check can be performed as follows: the distance between two particles divided by the sum of their radii should be more than 50 . Otherwise in Sec. 12.1 the major semi-axis value "a" should be increased using the coefficient $\mathrm{k}_{\mathrm{a}}$.

The problem of axisymmetric interaction of bodies was solved exactly in [1], [11], [12], [15]. Here, the peripheral bodies move along ellipses. In item 14.12.6 those orbits are calculated, and they are plotted in coordinates ynb(xnb). The index $i 2=1,2 . \mathrm{N}$ in MathCad can be used to call for plotting graphs not for all particles. For instance, the orbits will to be plotted for each third particle at the index $i 2=1,3$. N. Those graphs confirm that the set configuration of interacting particles is indeed axisymmetric, and the particles move in an elliptic orbit with the required eccentricity $e$.

In item 14.12.7, the orbit plane rotates through an angle $\varepsilon$ relative to the axis $\mathrm{x}$. Here, differential equations (5) «work» along all the three axes, x, y, and $z$, despite the fact that the initial statement of the problem was planar. Due to the latter rotation, to 20 trustworthiness criterions considered in $[18,19]$, one more criterion can 
be added. If integration of Eqs. (5) yields orbits deflected from the initial plane, then the deviations should be identified as inaccuracies of the integration method.

In item 14.12.8, we pass to non-dimensional variables. Here, $\mathrm{ms}_{\mathrm{N}+1}$ is the sum of the masses of all the particles involved in the system, Am is the scaling length factor in meters, and $\mathrm{k}_{\mathrm{t}}$ and $\mathrm{k}_{\mathrm{v}}$ are the scaling time and velocity coefficients expressed in SI units. Those parameters were described in Sec. 3.

In the latter example, the scaling factor $\mathrm{Am}$ is calculated from the condition that the non-dimensional time unit should be equal to $P_{m}=1 \cdot 10^{-15} \mathrm{sec}$. Since Am is determined with some non-zero inaccuracy due to extraction of a cubic root, then a procedure for refining the quantity Am is to be applied.

It should be noted that ratio $2 \cdot \mathrm{a} / \mathrm{Am}=3.23$ for given unit of time $P_{m}$, i.e. the order is close to 1 . In this case the scaling factor Am is a characteristic size of a considered system, namely an average size of orbit $2 \cdot a$.

In item 14.12.9, according to formulas (4)-(5) [1] the variables are reduced to the center of mass of the system. Here, for instance, $\mathrm{Xc}_{\mathrm{N}+1}$ and $\mathrm{Vxc}_{\mathrm{N}+1}$ are the coordinate and the velocity of the center of mass. In the problem of interest, initially the center of mass is at the origin, so that the above values are both close to zero. Nonetheless, the procedure should be accomplished, and the parameter values of the center of mass, examined. The latter examination is a check of the algorithm for calculating initial conditions. In the present case, the values of the parameters are within $5 \cdot 10^{-18}$. That is why for performing Galactica calculations with a lower error preparation of initial conditions with a better accuracy is required. For instance, it would be desirable that the initial conditions be prepared with a larger number length than it can be made in MathCad.

In item 14.12.10, projections of the angular momentum $\mathrm{Mx}_{\mathrm{N}+1}, \mathrm{My}_{\mathrm{N}+1}$ and $\mathrm{Mz}_{\mathrm{N}+1}$ of the whole particle system and the absolute value of the angular momentum M0 are calculated. For the problem of interest, the total angular momentum of the whole system M00 can also be calculated by formula (16) [1]. Then, the check is to be performed. The sum of non-dimensional masses of the system should equal unity. We determine the relative error of the angular momentum M0 and its value M00 calculated by formula (16) [1], and also the relative inaccuracy of the scaling factors $A m, k_{t}$ and $k_{v}$. Those checks enable evaluation of the inaccuracy of the algorithm, and also the value of the computational inaccuracy. As it is seen, here the residual of the scaling factors is zero. This result was obtained through refinement of Am in item 14.12.8.

In item 14.12.11, we calculate the number $\mathrm{Mu}$ to be used in the input file galacf26.dat for specifying initial conditions for space-distributed substance. Here, the parameter $\mathrm{Mu}$ is not used. However, for right representation of particles in graphic mode this number should be made consistent with the number of particles $\mathrm{Nb}$. The table below exemplifies matched parameters for different values of $\mathrm{Nb}$ :

\begin{tabular}{|c|c|c|c|}
\hline $\mathrm{B}$ & $\mathrm{C}$ & $\mathrm{Mu}$ & $\mathrm{Nb}$ \\
\hline 0.5 & 0.25 & 4 & 30 \\
\hline 0.9 & 0.6 & 7 & 300 \\
\hline 0.9 & 0.7955 & 15 & 3000 \\
\hline
\end{tabular}

In item 14.12.12, initial data and conditions are written in a prn-file, for instance, axsyk109.prn. The file contains 16 columns. The first two lines contain general data involving 25 parameters. That is why one zero at the end of the first line is to be removed, and six zeroes at the end of the second line are to be removed, and the file is to be renamed in a dat-file, for instance, axsyk109.dat.

After the first two lines, additional lines, each comprising 16 values, appear. The total number of the lines is equal to the total number of the particles involved in the problem. At the end, there appears an information line, whose first number is the date of creation of the initial condition file: year.mm.dd.

The next two graphs show the coordinates and velocities in the particle system. Those values enable a check of the sought configuration planned in the problem statement. As it is seen from the graphs, the coordinates and velocities of the particles form an axisymmetric pattern; in other words, they correspond to the problem of axisymmetric interaction of the particles.

The above-considered program InCnPrpr.med was used to generate the following files: axsyk175.dat and axsyk473.dat for 175 and 473 particles, respectively. Those files permit check of operability of the exe-files, evaluation of the total errors in solving the problem, and integration step adjustment ensuring a required accuracy of problem solution. These axisymmetric configurations are noticeable by the maximal force of attraction between a peripheral particle and the central particle for the 175 system particles, and minimal - for the 473 system particles. If there are more particles in configuration, that peripheral particles are rejecting from the center.

Item 14.12.13 gives an algorithm for reading out an initial condition file or an integration data file of type garek29.dat. This becomes needed in setting initial conditions from a calculated data file of an analogous problem. Besides, that algorithm can be used for analyzing the output data.

In the first line, the number of bodies Nbg and index lengths ig and ig2 are defined. Here, instead of the operator READPRN, the operator READ is used. The latter operator permits reading-out of a non-ordered file. After the reading, required quantities can be displayed as shown in the graph.

Item 14.12.14 gives changes for files generation of problems on interaction of two, 175 and 473 particles.

\section{CONCLUSIONS}

The rapid development of science has led to a huge number of views. They are not digested by society and are not prepared to teach the new generation. This has led 
to a crisis in science education [20]. The way out of this crisis may be attracting able students for independent scientific research with the help of distance education [21]. For this purpose, we are developing a system Galactica, as well as other systems: http://www.smul1.newmail.ru/Systems_eng.html. To describe the movements and interactions we use the primary concepts: the coordinates, velocity, acceleration and force. We try not to use secondary abstract concepts: potential, action, Hamiltonian function, etc., for digesting of which requires a long course of study. An important part of research is data mining [22]. Currently, most of the methods for data mining associated with their probabilistic interpretation. In the proposed systems we develop such data mining, which contributes to a deterministic understanding of the phenomena.

The executed investigations of axisymmetric model of the atom with the help of system Galactica [14] discovered a number of phenomena that a new explains the behavior of atoms. Presented methods allow determining the initial conditions for the other models of the atom. Their study will establish a real mechanism of atom. In subsequent by consideration of interactions of atoms the mechanism of a molecule can be established. Further research along this path will gradually open the deterministic view of the micro-world.

We hope that the paper contains the necessary information that will allow even the novice researchers to use the system Galactica in their work. The above studies, students can perform in the process of learning. Those who carried away by these tasks by the end of training can get results that will enrich our understanding of the world.

\section{ACKNOWLEDGMENT}

Long-term calculations at developing module of the system Galactica with Coulomb interaction were performed on the supercomputers of the Siberian Supercomputing Centre in the ICMMG SB RAS, Novosibirsk, Russia.

\section{REFERENCES}

[1] J.J. Smulsky, "The System of Free Access Galactica to Compute Interactions of N-Bodies," I.J. Modern Education and Computer Science, 2012, Vol.4, 11, 1-20. http://www.mecs-press.org/, http://dx.doi.org/10.5815/ijmecs.2012.11.01.

[2] J.J. Smulsky, System Galactica, Institute of Earth's Cryosphere SB RAS, Tyumen, 2012. http://www.ikz.ru/ smulski/GalactcW/.

[3] A.D. Vlasov, Classical Direction in Quantum Mechanics, Moscow: Moscow Radio Engineering Institute, 1993, 229 p. (In Russian).

[4] Ph.M. Kanarev, "The Spectrum of the Universe," Galilean Electrodynamics, 2009, Vol. 20, Special Issues 1, 13-17.

[5] Ph.M. Kanarev, Monograph Microcosm. New knowledge in the fundamental sciences, Saarbrucken, Germany: "Palmarium Academic Publishing", 2013, 588 p. ISBN 378-3-8473-9514-0. (In Russian).
[6] M. Gryziński, "Spin-Dynamical Theory of the WaveCorpuscular Duality," International Journal of Theoretical Physics, 1987, Vol. 26, No. 10, 967-980. http://dx.doi.org/10.1007/BF00670821.

[7] M. Gryziński, "Classical Theory of Atomic Collisions. I. Theory of Inelastic Collisions," Physical Review A, 1965, Vol. 138, No. 2A, 336-358. http://dx.doi.org/10.1103/PhysRev.138.A336.

[8] M. Gryziński, "Ramsauer Effect as a Result of the Dynamic Structure of the Atomic Shell," Physical Review Letters, 1970, Vol. 24, No. 2, 45-47. http://dx.doi.org/10.1103/PhysRevLett.24.45.

[9] M. Gryziński, "Classical Theory of Electronic and Ionic Inelastic Collisions," Physical Review, 1959, Vol. 115, No. 2 , http://dx.doi.org/10.1103/PhysRev.115.374.

[10] M. Gryziński, "Stopping Power of a Medium for Heavy, Charged Particles," Physical Review A, 1957, Vol. 107, No. 6, 1471-1475. http://dx.doi.org/10.1103/PhysRev.107.1471.

[11] J.J. Smulsky, The Theory of Interaction, Publishing House of Novosibirsk University, Novosibirsk, 1999. (in Russian) http://www.ikz.ru/ smulski/TVfulA5_2.pdf.

[12] J.J. Smulsky, The Theory of Interaction, Publishing house Cultural Information Bank, Ekaterinburg, 2004. (in English) http://www.ikz.ru/ smulski/TVEnA5_2.pdf.

[13] J.J. Smulsky, Electrodynamics of moving bodies. Determination of forces and calculation of movements, Sarbrucken, Germany: "Palmarium Academic Publishing", 2014, 324 p. ISBN 978-3-659-98421-1. (In Russian). For information see http://www.ikz.ru/ smulski/Papers/InfElMvBEn.pdf.

[14] J.J. Smulsky, Axisymmetric Coulomb Interaction and Instability of Orbits, Institute of the Earth Cryosphere SB RAS, Tyumen, 2013, 30 p., Fig.: 12, Refer: 22, Russian, Dep. In VINITI 28.10.2013, No. 304-V2013. (In Russian) http://www.ikz.ru/ smulski/Papers/KulInt2.pdf.

[15] J.J. Smulsky, "Axisymmetrical problem of gravitational interaction of N-bodies," Mathematical modeling, Vol. 15, No 5, 2003, 27-36. (In Russian http://www.smull.newmail.ru/Russian1/IntSunSyst/ Osvnb4.doc).

[16] I. I. Smul'skii, "Multilayer Ring Structures," Physics of Particles and Nuclei Letters, Vol. 8, No. 5, 2011, 436-440. http://dx.doi.org/10.1134/S1547477111050189. http://www.ikz.ru/ smulski/Papers/PHPL436.pdf.

[17] B.M. Yavorsky and A.A. Detlaf, Handbook of Physics. For Engineers and Students. Fourth edition, revised, Moscow: Publishing House "Science". Main Edition of Physical and Mathematical literature, 1968, $940 \mathrm{p}$.

[18] V. P. Melnikov and J. J. Smulsky, Astronomical Theory of Ice Ages: New Approximations. Solutions and Challenges, Academic Publishing House, Novosibirsk, 2009. http://www.ikz.ru/ smulski/Papers/AsThAnE.pdf.

[19] E. A. Grebenikov and J. J. Smulsky, Numerical Investigation of the Mars Orbit Evolution in the Time Interval of Hundred Million, A. A. Dorodnitsyn Computing Center of RAS, Moscow, 2007. (in Russian http://www.ikz.ru/ smulski/Papers/EvMa100m4t2.p df).

[20] T. Wibig and P. Dam-o, "The Model of the Evolution of the Knowledge Content and Contemporary Science Education Crisis," I.J. Modern Education and Computer Science, 2012, 61-75. http://dx.doi.org/10.5815/ijmecs.2012.01.08. 
[21] M. Bogdanović, "Growing Importance of Distance Education," I.J. Modern Education and Computer Science, 2012, 3, 35-41. http://dx.doi.org/10.5815/ijmecs.2012.03.05.

[22] Al M. Sakha'a and A. Malibari, "Improve Teaching Method of Data Mining Course," I.J. Modern Education and Computer Science, 2012, 2, 15-22. http://dx.doi.org/10.5815/ijmecs.2012.02.03.

\section{Authors' Profiles}

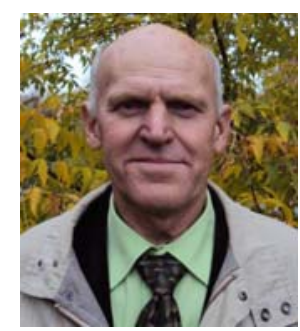

Joseph J. Smulsky was born in 1944 in the village of Poninka, Khmelnytsky region, the Republic of Ukraine, the Soviet Union. In 1967 he received the diploma in mechanical engineering after defending his graduation project "Space Elevator" at the Aviation Institute, Kharkov. In 1980 he received the degree of candidate of technical sciences after defending his dissertation "Investigation of the Hydrodynamics of the Vortex Chambers" at the Institute of Thermophysics SB AS USSR, Novosibirsk. In 1993 he received the degree of Doctor of Physical and Mathematical Sciences after defending his dissertation "Aerodynamics and Pprocesses in the Vortices". In 2006 he was to given the title of Professor in the Department of Theoretical and Applied Mechanics.

Prof. Joseph J. Smulsky from 1967 to 1988 worked in Research Institutes and Design bureaus of Novosibirsk. Since 1988 he has been working as chief scientist of the Institute of Earth Cryosphere. He is the author and the co-author more than 150 scientific works and inventions, including five monographs. His research interests include a wide range of problems: foundations of mechanics and physics, vortex flow, air pollution, wind energy, global climate changes etc http://www.smull.newmail.ru/, some of which are presented in recent publications:

1. Smulsky J.J. and Smulsky Ya.J. Dynamic Problems of the Planets and Asteroids, and Their Discussion. International Journal of Astronomy and Astrophysics, Vol. 2, No. 3, 2012, pp. 129-155. http://dx.doi:10.4236/ijaa.2012.23018.

2. Smulsky J.J. Electrodynamics of moving bodies. Determination of forces and calculation of movements. Saarbrucken, Germany: "Palmarium Academic Publishing”, 2014. 324 p. ISBN 978-3-659-98421-1.

3. Smulsky J.J. and Krotov O.I. New Computing Algorithm of the Earth's Insolation. Applied Physics Research, Vol. 6, No. $\quad 4 ; \quad 2014, \quad$ p. 56-82. http://dx.doi.org/10.5539/apr.v6n4p56. 
Appendix 1. Example of Mathcad program for generating an initial condition file for Coulomb's interaction

\title{
InCnPrClb.mcd . Example of calculation of the initial conditions for the program Galactica at Coulomb's interactions. CompletedD3.09.2012, modified 17.09.2014
}

\author{
14.12.1. Number of bodies
}
$\mathrm{Nb}:=9$
$\mathrm{N}:=\mathrm{Nb}-1 \quad \mathrm{i}:=0 \ldots \mathrm{N} \quad \mathrm{i} 1:=1 \ldots \mathrm{N}$
$\mathrm{N}=8$

14. 12.2. Constants

From Handbook by Yavorsky \& Detlaf: pp.749, 910, 912, 913.

$$
\begin{aligned}
& \mathrm{m}_{\mathrm{e}}:=9.1091 \cdot 10^{-31} \quad \mathrm{~m}_{\mathrm{p}}:=1.67252 \cdot 10^{-27} \mathrm{~m}_{\mathrm{ne}}:=1.67482 \cdot 10^{-27} \quad \mathrm{e}_{\mathrm{e}}:=4.80298 \cdot 10^{-10} \\
& \mathrm{R} 0:=1.5 \cdot 10^{-15} \quad \mathrm{R}_{\mathrm{e}}:=1.5 \cdot 10^{-15} \quad \mathrm{a}_{\mathrm{B} 0}:=5.29167 \cdot 10^{-11} \quad \varepsilon \mathrm{d}:=1 \quad \mathrm{a}_{\mathrm{k}}:=1
\end{aligned}
$$

14.12.3. Properties of bodies and of their motions

$$
\begin{aligned}
& \mathrm{A}_{\mathrm{N}}:=16 \quad \mathrm{Z}_{\mathrm{p}}:=8 \quad \mathrm{n}_{\mathrm{n}}:=2 \quad \mathrm{l}_{\mathrm{n}}:=1 \quad \mathrm{r}_{\mathrm{m}}:=\mathrm{a} \mathrm{B} 0 \cdot \frac{\left[3 \cdot \mathrm{n}_{\mathrm{n}}^{2}-1_{\mathrm{n}} \cdot\left(1_{\mathrm{n}}+1\right)\right]}{2 \cdot \mathrm{Z}_{\mathrm{p}}} \quad \mathrm{r}_{\mathrm{n}}:=\mathrm{R} 0 \cdot \mathrm{A}_{\mathrm{N}}{ }^{\frac{1}{3}} \\
& \mathrm{a}:=\mathrm{r}_{\mathrm{m}} \cdot \mathrm{a} \mathrm{k} \quad \mathrm{e}:=0.15 \quad \varepsilon 0:=0.409 \quad \mathrm{a}=3.30729375 \cdot 10^{-11} \quad \mathrm{r}_{\mathrm{n}}=3.77976314968462 \cdot 10^{-15}
\end{aligned}
$$

14. 12.4. Masses of bodies in $\mathrm{kg}$ and their radiuses in $\mathrm{m}$

$$
\mathrm{Z}_{\mathrm{n}}:=\mathrm{A}_{\mathrm{N}}-\mathrm{Z}_{\mathrm{p}} \quad \mathrm{m}_{0}:=\mathrm{Z}_{\mathrm{p}} \cdot \mathrm{m}_{\mathrm{p}}+\mathrm{Z}_{\mathrm{n}} \cdot \mathrm{m}_{\mathrm{ne}} \quad \mathrm{m}_{\mathrm{i} 1}:=\mathrm{m}_{\mathrm{e}} \quad \operatorname{ram}_{0}:=\mathrm{r}_{\mathrm{n}} \quad \quad \operatorname{ram}_{\mathrm{i} 1}:=\mathrm{R}_{\mathrm{e}}
$$

14. 12.5. Coordinates and velocities of bodies acordindly to:

nulsky J.J. Axisymmetrical problem of gravitational interaction of N-bodies // Mathematical modelling. 2003, Vol. 15, No 5, Pp. 27-36. (In Russian http://www.smul1.newmail.ru/Russian1/IntSunSyst/Osvnb4.doc).

For two bodies interactions see item 14. 12.14.

$$
\begin{aligned}
& \alpha_{1}:=-\frac{1}{1+\mathrm{e}} \quad \mathrm{Rp}:=\mathrm{a} \cdot \frac{2 \cdot \alpha_{1}+1}{\alpha_{1}} \quad \mathrm{f}_{\mathrm{N}}:=0.25 \cdot \sum_{\mathrm{i} 2=2}^{\mathrm{N}} \frac{1}{\sin \left[\frac{(\mathrm{i} 2-1) \cdot \pi}{\mathrm{N}}\right]} \\
& \mu 1:=-\frac{10^{-9} \cdot \mathrm{e}^{2} \mathrm{e}^{2}}{\mathrm{~m}_{1} \cdot \varepsilon \mathrm{d}} \cdot\left(\mathrm{N}-\mathrm{f}_{\mathrm{N}}\right) \\
& \mathrm{vp}:=\sqrt{\frac{\mu 1}{\alpha_{1} \cdot \mathrm{Rp}} \quad \mathrm{d} \phi:=\frac{2 \cdot \pi}{\mathrm{N}} \quad \phi 0_{\mathrm{i} 1}:=(\mathrm{i} 1-1) \cdot \mathrm{d} \phi} \quad \alpha_{1}=-0.869565217391304
\end{aligned}
$$

Coordinates and velocities in the plane of orbits, in $\mathrm{m}$ and $\mathrm{m} / \mathrm{sec}$

$\mathrm{xo}_{\mathrm{i} 1}:=\mathrm{Rp} \cdot \cos \left(\phi 0_{\mathrm{i} 1}\right) \quad \mathrm{yo}_{\mathrm{i} 1}:=\mathrm{Rp} \cdot \sin \left(\phi 0_{\mathrm{i} 1}\right) \quad \mathrm{zo}_{\mathrm{i} 1}:=0 \quad \mu 1=-1.315657523093202 \cdot 10^{3}$ 
Appendix 1 (continued).

$\mathrm{vxo}_{11}:=-\mathrm{vp} \cdot \sin \left(\phi 0_{\mathrm{i} 1}\right) \quad \mathrm{vyo}_{11}:=\mathrm{vp} \cdot \cos \left(\phi 0_{\mathrm{i} 1}\right) \quad \mathrm{vzo}_{11}:=0 \quad \mathrm{f}_{\mathrm{N}}=2.804865846209121$

Checking the distanses between bodies. If $d$ is less than $\mathbf{5 0}$, it is need to increase $a$ $\mathrm{do}:=\sqrt{\left(\mathrm{xo}_{2}-\mathrm{xo}_{1}\right)^{2}+\left(\mathrm{yo}_{2}-\mathrm{yo}_{1}\right)^{2}+\left(\mathrm{zo}_{2}-\mathrm{zo}_{1}\right)^{2}} \mathrm{~d}:=\frac{\mathrm{do}}{\left(2 \cdot \mathrm{ram}_{1}\right)} \quad \mathrm{d}=7.17199696984111710^{3}$

14.12.6. The exact motion of bodies. Checking the planned configuration of th problem

$$
\begin{aligned}
& \mathrm{J}:=50 \quad \phi_{0}:=0 \quad \mathrm{j}:=1 \ldots \mathrm{J} \quad \phi_{\mathrm{j}}:=\phi_{\mathrm{j}-1}+\frac{2 \cdot \pi}{\mathrm{J}} \\
& \operatorname{rnb}_{\mathrm{i} 1, \mathrm{j}}:=\frac{\mathrm{Rp}}{\left[\left(\alpha_{1}+1\right) \cdot \cos \left(\phi_{\mathrm{j}}-\phi 0_{\mathrm{i} 1}\right)-\alpha_{1}\right]} \quad \mathrm{Ra}:=\frac{\mathrm{Rp}}{\left[-\left(\alpha_{1}+1\right)-\alpha_{1}\right]} \quad \mathrm{D}_{\mathrm{M}}:=1.1 \cdot \mathrm{Ra} \\
& \mathrm{xnb}_{11, \mathrm{j}}:=\mathrm{rnb}_{11, \mathrm{j}} \cdot \cos \left(\phi_{\mathrm{j}}\right) \quad \mathrm{ynb}_{11, \mathrm{j}}:=\mathrm{rnb}_{\mathrm{i} 1, \mathrm{j}} \cdot \sin \phi_{\mathrm{j}} \quad \mathrm{i} 2:=1,2 . . \mathrm{N}
\end{aligned}
$$

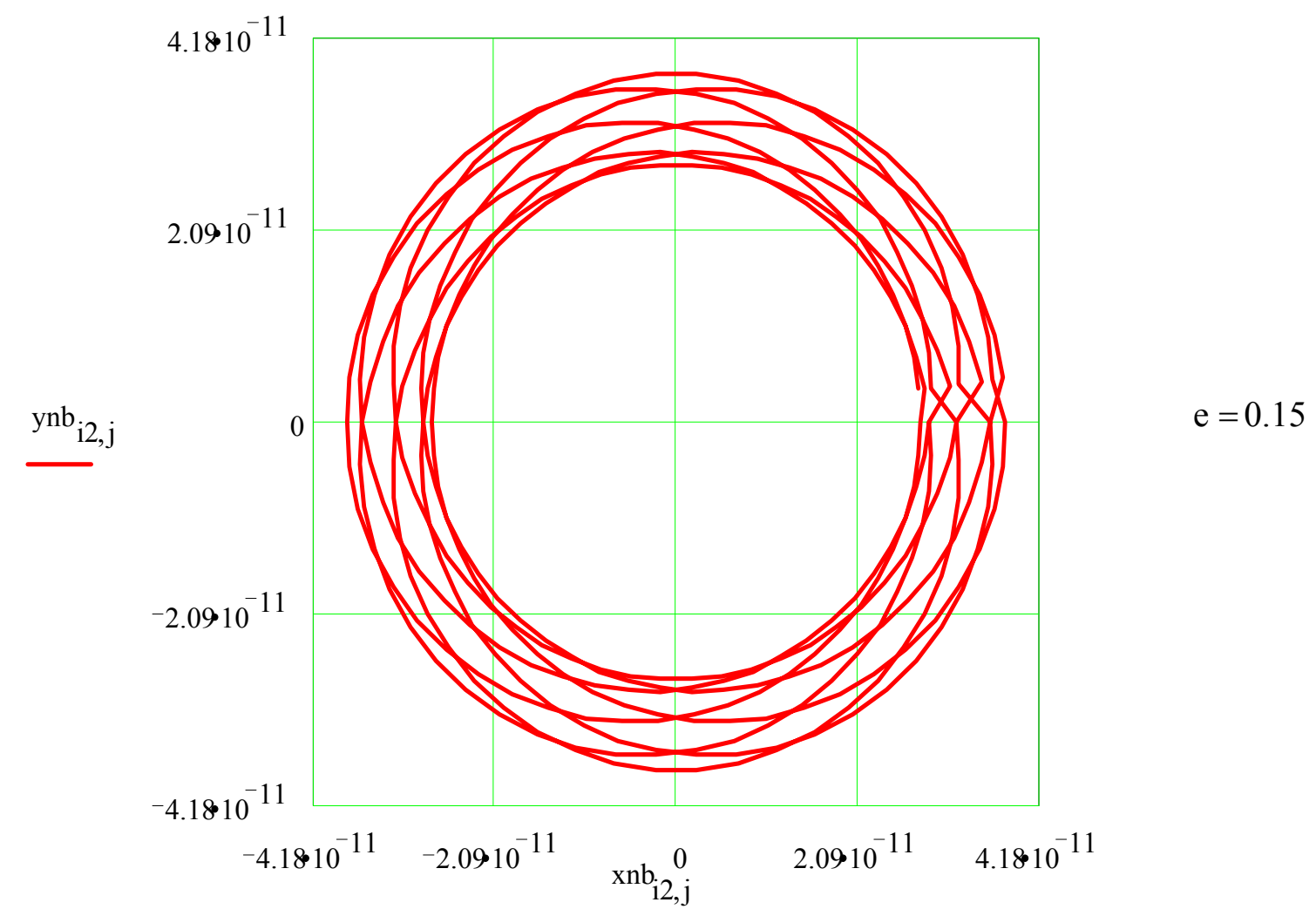

14.12.7. Coordinates and velocities in the equatorial plane

$$
\begin{aligned}
& \mathrm{xm}_{11}:=\mathrm{xo}_{\mathrm{i} 1} \quad \mathrm{ym}_{11}:=\mathrm{yo}_{\mathrm{i} 1} \cdot \cos (\varepsilon 0)-\mathrm{zo}_{\mathrm{i} 1} \cdot \sin (\varepsilon 0) \quad \mathrm{zm}_{11}:=\mathrm{yo}_{\mathrm{i} 1} \cdot \sin (\varepsilon 0)+\mathrm{zo}_{\mathrm{i} 1} \cdot \cos (\varepsilon 0) \\
& \operatorname{vxms}_{11}:=\operatorname{vxo}_{11} \operatorname{vyms}_{11}:=\operatorname{vyo}_{11} \cdot \cos (\varepsilon 0)-\operatorname{vzo}_{11} \cdot \sin (\varepsilon 0) \quad \mathrm{vzms}_{11}:=\mathrm{vyO}_{11} \cdot \sin (\varepsilon 0)+\mathrm{vzo}_{11} \cdot \cos (\varepsilon 0) \\
& \mathrm{xm}_{\mathrm{f}}:=0 \quad \mathrm{ym}_{\mathrm{f}}:=0 \quad \mathrm{zm}_{\mathrm{f}}:=0 \quad \text { vxmg }:=0 \quad \text { vymg }:=0 \quad \text { vzmg }:=0
\end{aligned}
$$


14.12.8. The transition to the dimensionless variables

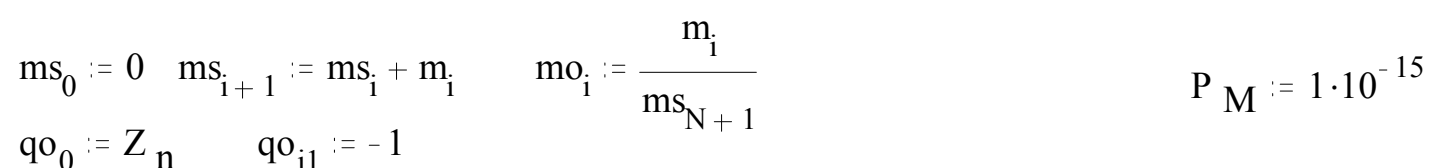

Determination of the length scale that the dimensionless time unit is equel one $10^{\wedge}-15$ of seco $\mathrm{k}_{\mathrm{t}}:=\frac{1}{\mathrm{P}_{\mathrm{M}}} \quad \mathrm{Am}:=\left[\frac{\left(10^{-9} \cdot \mathrm{e}_{\mathrm{e}}^{2}\right)}{\varepsilon \mathrm{d} \cdot \mathrm{ms}_{\mathrm{N}+1} \cdot \mathrm{k}_{\mathrm{t}}^{2}}\right]^{\frac{1}{3}} \quad \mathrm{Am}=2.049767130503777 \cdot 10^{-11}$

\section{Clarification of Am}

$$
\begin{aligned}
& \mathrm{Am}:=2.049767130503774 \cdot 10^{-11} \quad \mathrm{k}_{\mathrm{v}}:=\sqrt{\frac{\varepsilon \mathrm{d} \cdot \mathrm{ms}_{\mathrm{N}+1} \cdot \mathrm{Am}}{10^{-9} \cdot \mathrm{e}^{2}}} \quad \mathrm{Am}:=\frac{1}{\mathrm{k}_{\mathrm{t}} \cdot \mathrm{k}_{\mathrm{v}}} \\
& \mathrm{x}_{\mathrm{i}}:=\frac{\mathrm{xm}_{\mathrm{i}}}{\mathrm{Am}} \quad \mathrm{y}_{\mathrm{i}}:=\frac{\mathrm{ym}_{\mathrm{i}}}{\mathrm{Am}} \quad \mathrm{z}_{\mathrm{i}}:=\frac{\mathrm{zm}_{\mathrm{i}}}{\mathrm{Am}} \quad \mathrm{ra}_{\mathrm{i}}:=\frac{\mathrm{ram}_{\mathrm{i}}}{\mathrm{Am}} \quad \mathrm{Am}=2.049767130503774 \cdot 10^{-11} \\
& \mathrm{vx}_{\mathrm{i}}:=\mathrm{vxms}_{\mathrm{i}} \cdot \mathrm{k}_{\mathrm{v}} \quad \mathrm{vy}_{\mathrm{i}}:=\text { vyms s}_{\mathrm{i}} \cdot \mathrm{k}_{\mathrm{v}} \quad \mathrm{vz}_{\mathrm{i}}:=\mathrm{vzms}_{\mathrm{i}} \cdot \mathrm{k}_{\mathrm{v}}
\end{aligned}
$$

14.12.9. The center of mass of the interacting bodies

$$
\begin{aligned}
& \mathrm{Xc}_{0}:=0 \quad \mathrm{Yc}_{0}:=0 \quad \mathrm{Zc}_{0}:=0 \quad \mathrm{Vxc}_{0}:=0 \quad \mathrm{Vyc}_{0}:=0 \quad \mathrm{Vzc}_{0}:=0 \\
& \mathrm{Xc}_{\mathrm{i}+1}:=\mathrm{Xc}_{\mathrm{i}}+\mathrm{mo}_{\mathrm{i}} \cdot \mathrm{x}_{\mathrm{i}} \quad \mathrm{Yc}_{\mathrm{i}+1}:=\mathrm{Yc}_{\mathrm{i}}+\mathrm{mo}_{\mathrm{i}} \cdot \mathrm{y}_{\mathrm{i}} \quad \mathrm{Zc}_{\mathrm{i}+1}:=\mathrm{Zc}_{\mathrm{i}}+\mathrm{mo}_{\mathrm{i}} \cdot \mathrm{z}_{\mathrm{i}} \\
& \mathrm{Vxc}_{\mathrm{i}+1}:=\mathrm{Vxc}_{\mathrm{i}}+\mathrm{mo}_{\mathrm{i}} \cdot \mathrm{Vx}_{\mathrm{i}} \quad \mathrm{Vyc}_{\mathrm{i}+1}:=\mathrm{Vyc}_{\mathrm{i}}+\mathrm{mo}_{\mathrm{i}} \cdot \mathrm{vy}_{\mathrm{i}} \quad \mathrm{Vzc}_{\mathrm{i}+1}:=\mathrm{Vzc}_{\mathrm{i}}+\mathrm{mo}_{\mathrm{i}} \cdot \mathrm{vz}_{\mathrm{i}}
\end{aligned}
$$

\section{Coordinates and velocities relatively the center of mass}

$$
\begin{array}{lll}
\mathrm{x}_{\mathrm{i}}:=\mathrm{x}_{\mathrm{i}}-\mathrm{Xc}_{\mathrm{N}+1} & \mathrm{y}_{\mathrm{i}}:=\mathrm{y}_{\mathrm{i}}-\mathrm{Yc}_{\mathrm{N}+1} & \mathrm{z}_{\mathrm{i}}:=\mathrm{z}_{\mathrm{i}}-\mathrm{Zc}_{\mathrm{N}+1} \quad \mathrm{Xc}_{\mathrm{N}+1}=-2.032879073410321 \cdot 10^{-20} \\
\mathrm{vx}_{\mathrm{i}}:=\mathrm{vx}_{\mathrm{i}}-\mathrm{Vxc}_{\mathrm{N}+1} & \mathrm{vy}_{\mathrm{i}}:=\mathrm{vy}_{\mathrm{i}}-\mathrm{Vyc}_{\mathrm{N}+1} & \mathrm{vz}_{\mathrm{i}}:=\mathrm{vz}_{\mathrm{i}}-\mathrm{Vzc}_{\mathrm{N}+1} \quad \mathrm{Vxc}_{\mathrm{N}+1}=5.204170427930421 \cdot 10^{-18}
\end{array}
$$

14. 12. 10. Angular momentum of the system of bodies

$$
\begin{aligned}
& \mathrm{Mx}_{\mathrm{i}}:=0 \quad \mathrm{My}_{\mathrm{i}}:=0 \quad \mathrm{Mz}_{\mathrm{i}}:=0
\end{aligned}
$$

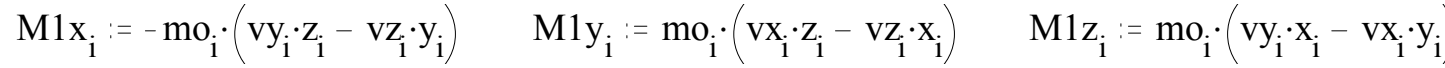

$$
\begin{aligned}
& \mathrm{Mx}_{\mathrm{i}+1}:=\left(\mathrm{Mx}_{\mathrm{i}}\right)+\mathrm{M} 1 \mathrm{x}_{\mathrm{i}} \quad \mathrm{My}_{\mathrm{i}+1}:=\left(\mathrm{My}_{\mathrm{i}}\right)+\mathrm{M} 1 \mathrm{y}_{\mathrm{i}} \quad \mathrm{Mz}_{\mathrm{i}+1}:=\left(\mathrm{Mz}_{\mathrm{i}}\right)+\mathrm{M} 1 \mathrm{z}_{\mathrm{i}} \\
& \mathrm{Mx}_{\mathrm{N}+1}=-9.665352161902836 \cdot 10^{-19} \quad \mathrm{My}_{\mathrm{N}+1}=-0.053108187925464 \\
& \mathrm{Mz}_{\mathrm{N}+1}=0.122526400051667
\end{aligned}
$$

\section{Verification}


Appendix 1 (continued)

$$
\begin{aligned}
& \mathrm{msr}_{0}:=0 \quad \mathrm{msr}_{\mathrm{i}+1}:=\mathrm{msr}_{\mathrm{i}}+\mathrm{mo}_{\mathrm{i}} \quad \mathrm{msr}_{\mathrm{N}+1}=1 \\
& \mathrm{M} 00:=\frac{\mathrm{N} \cdot \mathrm{mo}_{1} \cdot \mathrm{Rp} \cdot \mathrm{vp} \cdot \mathrm{k}_{\mathrm{V}}}{\mathrm{Am}} \quad \mathrm{M} 0:=\sqrt{\left(\mathrm{Mx}_{\mathrm{N}+1}\right)^{2}+\left(\mathrm{My}_{\mathrm{N}+1}\right)^{2}+\left(\mathrm{Mz}_{\mathrm{N}+1}\right)^{2}} \\
& \frac{\mathrm{M} 0-\mathrm{M} 00}{\mathrm{M} 0}=0 \quad\left(\mathrm{Am}-\frac{1}{\mathrm{k}_{\mathrm{t}} \cdot \mathrm{k}_{\mathrm{v}}} \cdot \frac{1}{\mathrm{Am}}=0\right.
\end{aligned}
$$

14.12.11. To coordinate the size of area with the number of bodies need to change the $\mathbf{B}<=\mathbf{1}$ and $\mathrm{C} 1<=1$ so that $\mathrm{Nbar}>=\mathrm{Nb}$.

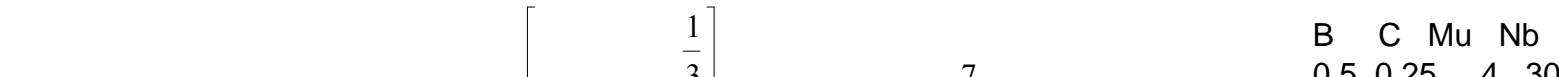

$$
\begin{aligned}
& \left.\mathrm{B}:=0.25 \mathrm{C} 1:=0.1 \quad \mathrm{Mu}:=\operatorname{ceil}(\mathrm{Nb}+1)^{\overline{3}}\right] \quad \mathrm{dT}:=1.0 \cdot 10^{-7} \quad \mathrm{Mu}=3 \quad \begin{array}{llll}
0.5 & 0.25 & 4 & 30
\end{array} \\
& \text { Nbar }:=(1+\mathrm{Mu}) \cdot(1+\mathrm{Mu} \cdot \mathrm{B}) \cdot(1+\mathrm{Mu} \cdot \mathrm{C} 1) \quad \mathrm{Nbar}=9.1 \quad \mathrm{Nb}=9 \quad 0.90 .7955153000
\end{aligned}
$$

\subsubsection{Writing data to the file name.prn}

$\mathrm{k}:=0 . .14 \quad 1:=7 . .12 \quad 12:=5 . .14 \quad \mathrm{R}_{0, \mathrm{k}}:=0 \quad \mathrm{R}_{0,7}:=\mathrm{Mx}_{\mathrm{N}+1} \quad \mathrm{R}_{0,8}:=\mathrm{My}_{\mathrm{N}+1}$

$\mathrm{R}_{0,9}:=\mathrm{Mz}_{\mathrm{N}+1} \quad \mathrm{R}_{1,0}:=0 \quad \mathrm{R}_{1,1}:=\mathrm{dT} \quad \mathrm{R}_{1,2}:=1 \quad \mathrm{R}_{1,3}:=1 \quad \mathrm{R}_{1,4}:=\mathrm{Nb}$

$\mathrm{R}_{1,5}:=1 \quad \mathrm{R}_{1,6}:=\mathrm{B} \quad \mathrm{R}_{1,7}:=\mathrm{C} 1 \quad \mathrm{R}_{1,8}:=\mathrm{Mu} \quad \mathrm{R}_{1,9}:=\varepsilon \mathrm{d} \quad \mathrm{R}_{\mathrm{i}+2,0}:=\mathrm{mo}_{\mathrm{i}} \quad \mathrm{R}_{\mathrm{i}+2,1}:=\mathrm{x}_{\mathrm{i}}$

$\mathrm{R}_{\mathrm{i}+2,2}:=\mathrm{y}_{\mathrm{i}} \quad \mathrm{R}_{\mathrm{i}+2,3}:=\mathrm{z}_{\mathrm{i}} \quad \mathrm{R}_{\mathrm{i}+2,4}:=\mathrm{vx}_{1} \quad \mathrm{R}_{\mathrm{i}+2,5}:=\mathrm{vy}_{\mathrm{i}} \quad \mathrm{R}_{\mathrm{i}+2,6}:=\mathrm{vz}_{1} \quad \mathrm{R}_{\mathrm{i}+2,1}:=0$

$\mathrm{R}_{\mathrm{i}+2,13}:=\mathrm{ra}_{\mathrm{i}} \quad \mathrm{R}_{\mathrm{i}+2,14}:=0 \quad \mathrm{R}_{\mathrm{i}+2,15}:=\mathrm{qo}_{\mathrm{i}}$

Information line at end of file $13:=\mathrm{Nb}+2$

$\mathrm{R}_{13,0}:=20120918 \quad \mathrm{R}_{13,1}:=\mathrm{Nb} \quad \mathrm{R}_{13,2}:=\mathrm{ms}_{\mathrm{N}+1} \quad \mathrm{R}_{13,3}:=A m \quad \mathrm{R}_{13,4}:=\mathrm{k}_{\mathrm{v}} \quad \mathrm{R}_{13,12}:=0$

$\begin{array}{llllll}\text { WwwwRITEPRN"axsyk109.prn)" := R } & \text { e } & 0.15 & 0 & 0.15 & 0.15\end{array}$

"axsyk109.prn'"axsykc09.prn" "axsyk175.prn" "axsyk473.prn"

It is necessary 1 zeroat the end of 1-nd linend 6 zero at the end of 2-nd line of file name.prn to remove and to renamæhe file into a name.dat.

Verification coordinates and velocities
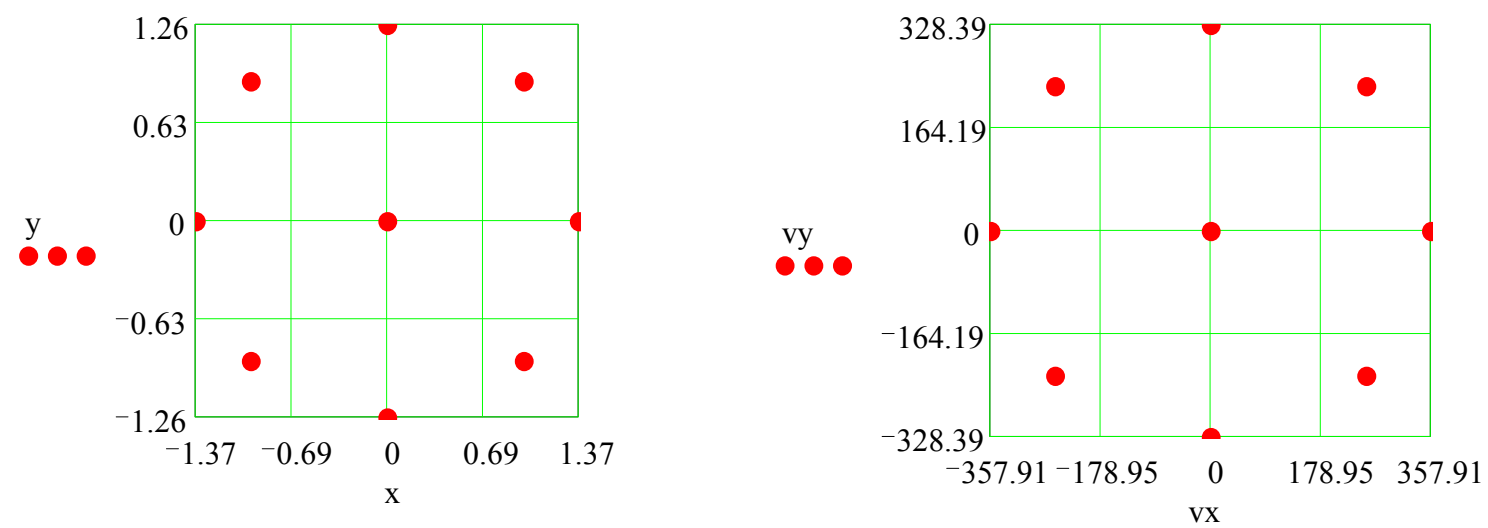


\section{Appendix 1 (end)}

\subsubsection{Reading the file of type garek29.dat}

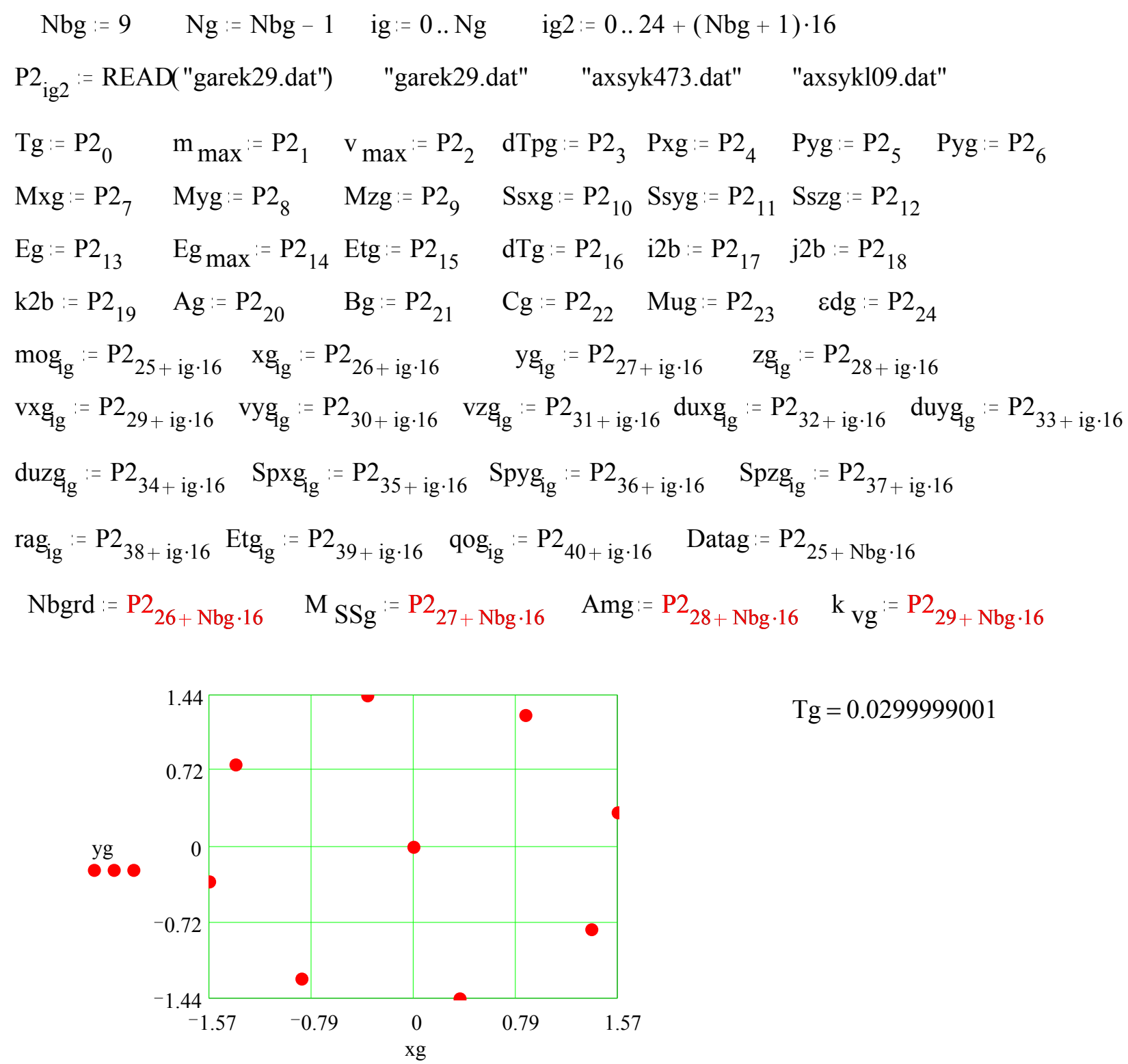

14.12.14. Change in items for $\mathrm{Nb}$-paricles interactions
$\mathrm{Nb}=2$
$\alpha_{1}:=-\frac{1}{1+\mathrm{e}} \quad \mathrm{Rp}:=\mathrm{a} \cdot \frac{2 \cdot \alpha_{1}+1}{\alpha_{1}}$
$\mathrm{m}_{\mathrm{pr}}:=\frac{\mathrm{m}_{0} \cdot \mathrm{m}_{1}}{\mathrm{~m}_{0}+\mathrm{m}_{1}}$
$\mu 1:=-\left(\frac{10^{-9} \cdot \mathrm{e}^{2}}{\mathrm{~m}_{\mathrm{pr}} \cdot \varepsilon \mathrm{d}}\right)$
$\mathrm{Nb}=175 \quad \mathrm{AN}=348 \quad \mathrm{Zp}=174 \quad \mathrm{nn}=174 \quad \mathrm{PM}=2^{\star} 10^{\wedge}-10 \quad \mathrm{~B}=0.8 \quad \mathrm{C} 1=0.55$
$\mathrm{Nb}=473 \quad \mathrm{AN}=946 \quad \mathrm{Zp}=472 \quad \mathrm{nn}=472 \quad \mathrm{PM}=2^{\star} 10^{\wedge}-9 \quad \mathrm{~B}=0.9 \quad \mathrm{C} 1=0.676$ 